\title{
Experimental Methodology for Measuring Combustion and Injection-Coupled Responses
}

\author{
Ryan C. Cavitt ${ }^{*}$, Robert A. Frederick, Jr. ${ }^{\dagger}$, and Vladimir G. Bazarov ${ }^{\dagger}$ \\ UAH Propulsion Research Center, Huntsville, Alabama, 35899
}

\begin{abstract}
A Russian scaling methodology for liquid rocket engines utilizing a single, full scale element is reviewed. The scaling methodology exploits the supercritical phase of the full scale propellants to simplify scaling requirements. Many assumptions are utilized in the derivation of the scaling criteria. A test apparatus design is presented to implement the Russian methodology and consequently verify the assumptions. This test apparatus will allow researchers to assess the usefulness of the scaling procedures and possibly enhance the methodology. A matrix of the apparatus capabilities for a RD-170 injector is also presented. Several methods to enhance the methodology have been generated through the design process.
\end{abstract}

$\begin{array}{ll}\mathrm{c} & =\text { Speed of sound } \\ \mathrm{D} & =\text { Diameter } \\ \mathrm{Eu} & =\text { Euler Number } \\ \mathbf{k} & =\text { Area ratio } \\ \mathrm{L} & =\text { Length } \\ n_{\mathrm{R}} & =\text { Mass flow rate } \\ \mathrm{Ma} & =\text { Mach Number } \\ \Delta \mathrm{P} & =\text { Pressure drop across injector } \\ \mathbf{R e} & =\text { Reynolds Number } \\ \rho & =\text { Density } \\ \mathbf{W} & =\text { Velocity }\end{array}$

\section{Nomenclature}

Subscripts

$F=$ fuel

fs $=$ full scale

$\mathrm{m}=$ model

ox $=$ oxidizer

$\Sigma=$ discharge

\section{Introduction}

$\mathrm{H}$ igh frequency combustion instability continues to hinder the development of liquid rocket engines (LRE). Unstable combustion is characterized by high amplitude chamber pressure fluctuations at distinct frequencies. In extreme cases, chamber pressure oscillations can lead to catastrophic loss of hardware. This destructive phenomenon can appear and destroy an engine in fractions of a second. ${ }^{1}$ Combustion instability is an interaction between the natural frequency of the engine hardware and the combustion process. Therefore, propellant injection is a critical component in the system. The complex processes of mixing, atomization and vaporization are proclaimed to be the leading processes responsible for instability.

Designing a LRE to circumvent this problem has proven to be a formidable task. The most straightforward approach to determine the stability of a LRE is to test the full scale engine. Unfortunately, this is usually not a feasible or efficient approach. Full scale testing is extremely expensive and time consuming. If full scale testing is to be used solely, the LRE designer is forced to rely completely on previous designs and experience for the initial design. In the event of unstable operation, modifications are required. Passive suppression methods such as baffles and resonators are commonly employed, but can prove insufficient. In this case, more extreme changes can be necessary at the cost of excessive time and resources. Engine stability considerations must be implemented early in

\footnotetext{
* Graduate Research Assistant, UAH Propulsion Research Center, 5000 Technology Drive, and AIAA Member.

+ Assistant Director, UAH Propulsion Research Center, 5000 Technology Drive, and AIAA Associate Fellow.

¥ Visiting Eminent Scholar of Propulsion, UAH Propulsion Research Center, 5000 Technology Drive, and AIAA Member.
} 
the design process to avoid modifying designed hardware to obtain stable combustion. Subscale testing can be implemented to avoid such a problematic cycle. A successful subscale experimental program will never substitute for full scale testing, but can aid in reducing the chances of experiencing high frequency combustion instability in the final design. Scaling can be the solution to stability characterization. Scaling procedures were heavily developed in the 1950's. ${ }^{2,3}$ Many scaling techniques are based on the dimensionless analysis of fluid dynamic and combustion criteria such as Reynolds number, Prandtl number and Damköhler number. To derive the scaling procedures, assumptions and limitations must be invoked. These assumptions and limitations make certain techniques incompatible with unique engines. Achieving similarity can be difficult or even impossible with an appropriate methodology in practice.

Many different approaches to subscale testing exist. Single element injector testing is obviously the simplest form of scaling. The use of only one injector limits the information that can be extracted from experiments. Intraelement phenomena are the only characteristics that can be represented by the single element technique. Injector flow and combustion characteristics are of key importance and can be taken from such testing. In the event of stable combustion at nominal operating conditions, stability rating can be used by varying operating conditions to identify the boundaries of instability and subsequently how far the operating conditions are from unstable operation. ${ }^{4}$ For instance, fuel mass flow rate can be throttled, changing the mixture ratio, to isolate feed system dynamic affects, also chamber pressure variations aid in scrutinizing atomization and vaporization affect.

With any scaling procedure there are limitations. The single injector method neglects interelement influences. The influence of neighboring sprays can have a significant affect on the overall stability of the system, which must be investigated with other means. Similarity matching are of utmost importance not only for the injector, but also the combustion chamber conditions. Scaling the full scale chamber conditions is not simple. Assumptions about the chamber conditions must be applied. The uniform temperature of the chamber is customarily taken as the temperature of the combustion products. This may not be an accurate assumption. The most dangerous modes of instability, from a catastrophic failure standpoint, are the tangential modes. In this instance, the maximum pressure fluctuations are occurring at the injector fire face where the temperature can be influenced by heat loss through the fire face and recirculation flow creating a complex temperature field. If the product temperature is used to determine the speed of sound of the full scale combustion chamber, an inaccurate condition is supplied to the model.

\section{Background}

The recent publication by Dexter, et al. ${ }^{4}$, has described a Russian methodology that remains to be tested in the U.S. The methodology was developed during the design of the RD-170 engine, which ultimately utilized a gasliquid injector. This technique takes advantage of the supercritical state of the propellants prior to injection in the full scale engine. A supercritical fluid is identified as a dense gas; therefore surface tension does not exist. This is the greatest simplification to enable successful scaling. The absence of surface tension indicates that the atomization and vaporization processes are not important for scaling. Modeling can be achieved without matching droplet similarity conditions such as Weber number and Ohnesorge number. This leaves mixing as the leading process in high frequency combustion instability to be scrutinized.

\section{A. Experimental Setup}

A schematic of the methodology experimental device can be seen in Fig. 1. A near atmospheric pressure, water cooled chamber is used to allow long duration firings. The chamber operates near atmospheric pressure following the proclamation that the mean chamber pressure has minimal influence on the chamber acoustic field spatial parameters. One of the advantages of the atmospheric pressure chamber is that the hardware can function and endure the pressure oscillation amplitude that will be excited for the duration of the test. A single full scale, bipropellant injector is placed near the chamber wall to excite tangential modes of combustion instability. For full scale engines with multiple injector designs residing in a single chamber, a representative cluster of bipropellant injectors can be substituted for the single injector. The injector or injector cluster is fired with gaseous propellants, which is a consequence of full scale supercritical propellants. The oxidizer is heated to change the thermodynamic properties entering the injector. A neutral gas can be introduced to aid in matching the discharge velocity of the oxidizer. In addition, the neutral gas will influence the thermodynamic properties of the mixture. A similar feed system is used for the fuel, with the absence of a heating element. The minimum data acquisition requirement is a high frequency pressure transducer monitoring pressure fluctuations in the combustion chamber.

The methodology publication lists many other scaling criteria. First, partial modeling must be applied. Choosing the correct similarity conditions is crucial to accurately represent the processes in the full scale engine. Second, 
sizing the chamber is required to match the transverse frequency. This includes the chamber dimensions and speeds of sound of the model and full scale engine. Lastly, two phases must be matched; the chamber acoustics and combustion process, as well as the manifold acoustics and injection process. The first involves the chamber acoustics, which have been addressed and the time delay for the reactants to convert to products. The second involves scaling the injector manifold geometry and propellant temperature to match the time for an acoustic disturbance to traverse the manifold.

The Korean Aerospace Research Institute (KARI) has implemented this Russian methodology to investigate recess ratio effects in double swirl injectors. ${ }^{5}$ The described test setup was assembled with a cluster of five injectors instead of a single injector. A scaling parameter $(\Pi)$ was developed to meet suite testing procedures and was implemented for the partial modeling criteria. The results of the study agree with common injector trends. As the recess ratio increases, mixing increases which increases combustion efficiency and instability. The successful use of this methodology beyond the original jet-swirl application is promising for use in other injector schemes.

\section{B. Testing Procedure}

An experimental procedure is suggested with this methodology description. A graphic representation can be seen in Fig. 2. The graph illustrates the following testing procedure for a single injector. The fuel mass flow rate is fixed and the oxidizer mass flow rate is smoothly varied to a maximum value and returned to the minimum. The chamber pressure is recorded continuously throughout this experiment. A new fuel mass flow rate is selected and the procedure is repeated until sufficient data is acquired surrounding the design point operating conditions. In order to use the testing procedure effectively, the scaled down design point operating conditions from the full scale engine must be determined prior to the creation of the test matrix. Lee, et al. ${ }^{5}$ suggest varying the oxidizer volumetric flow rate from one half to twice the oxidizer volumetric flow rate design point. To define the threshold between stable and unstable combustion, a pressure oscillation level must be set. Data analysis of the pressure fluctuations with a set limit of instability will define the boundary of instability at different operating conditions.

When utilizing this experiment during engine development, mapping the boundaries of instability can drive the design of injectors toward the stable regions. Ideally, the final design point would lie far from the instability region. This distance can be quantified via stability margin rating and used to compare different injector designs. Once a model injector design and operating conditions are finalized, the test injector is equivalent to the full scale injector and the subscale operating conditions can be extrapolated to the full scale conditions.

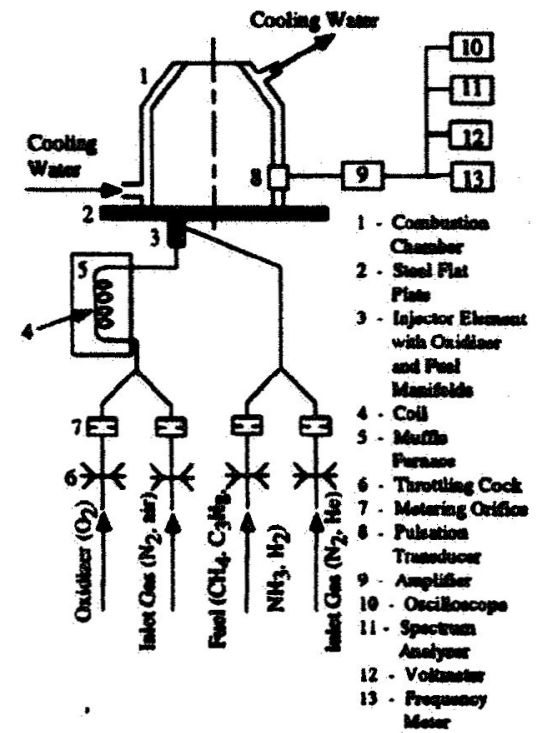

Figure 1. Russian Methodology Test Apparatus Schematic ${ }^{4}$

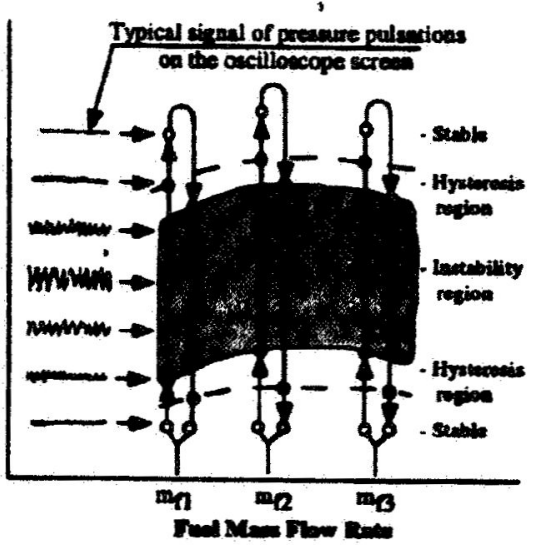

Figure 2. Boundary of Instability Testing Procedure ${ }^{4}$

\section{Approach}

\section{A. Objective}

The main objective of this project is to determine the feasibility of this simplistic Russian scaling technique. Determining the accuracy of the methodology could give LRE designers an invaluable tool to utilize throughout the engine development process. By verifying the scaling technique, strict adherence to the procedures can allow LRE designers to test multiple injector schemes and geometries and scale the test conditions up to full scale conditions for the final design. The assessment includes a scaling literature review, test apparatus design and experiments to verify the assumptions of the scaling procedures. Three injector schemes were selected for characterization; impinging jet, jet-swirl and swirl-swirl. A secondary objective of the project is to enhance the methodology, if possible 
improvements become evident. The inefficiencies or shortfalls of the scaling procedure will be exposed through the design process and experimental results.

\section{B. Injector Operating Conditions}

Since the methodology was created and implemented during the development of the RD-170 engine, the first injector to be tested is the injector from the main combustion chamber of this engine. Therefore the first set of tests will be to replicate the testing undergone by the Russian designers. The injector is a jet-swirl configuration with oxygen supplied axially and RP-1 introduced tangentially. The injector can be seen in Fig. 3.

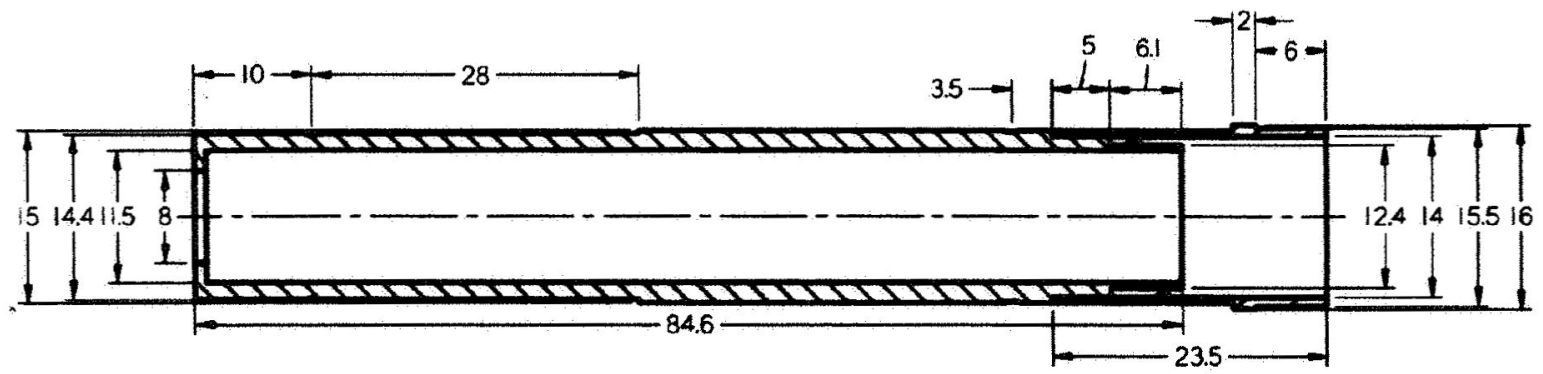

Figure 3. RD-170 Main Combustion Chamber Injector (all dimensions in mm)

The pertinent overall operating conditions for the RD-170 engine were determined by Manski, et al. ${ }^{6}$ A summary of the propellant conditions directly upstream of the injector manifold can be seen in Table 1 . These values were used to determine the full scale dimensionless parameters.

Table 1. RD-170 Main Combustion Chamber Operating Conditions

\begin{tabular}{|c|c|c|}
\hline & $\mathrm{R}$ & \\
\hline Critical Temperature [K] & 676 & 154.6 \\
\hline Critical Pressure [bar] & 21.7 & 50.4 \\
\hline Density [kg/m^3] & 807 & 155.38 \\
\hline Pressure [bar] & 278.26 & 278.26 \\
\hline Temperature [K] & 492.2 & 687.7 \\
\hline Total Mass Flow Rate [kg/s] & 158.05 & 440.7 \\
\hline Injector Average Mass Flow Rate [kg/s] & 0.583 & 1.626 \\
\hline Pressure Drop [bar] & 25.3 & 25.3 \\
\hline Viscosity [kg/m-s] & $3.237 \mathrm{E}-04$ & $4.001 \mathrm{E}-05$ \\
\hline Discharge Velocity [m/s] & 79.18 & 183.40 \\
\hline Speed of Sound [m/s] & 907.9 & 543.5 \\
\hline
\end{tabular}

Examination of Table 1 will reveal that the RP-1 is not above the critical temperature and therefore does not satisfy the supercritical phase criterion. The propellant is below the critical temperature by $183 \mathrm{~K}$. An assumption about the injector thermal environment and combustion chamber must be made to allow the methodology to be used in this particular engine. As the fuel swirls around the nozzle, the RP-1 will be heated through the interaction with the heated oxygen spray. Also, the time for the fuel to vaporize in the combustion chamber is assumed to be negligible compared to the time for mixing.

The main combustion chamber of the RD-170 engine uses multiple injector types. ${ }^{7}$ The U.S. patent application of this engine was referenced to verify the use of the overall operating conditions to a single injector. At least five different injector designs are employed in the engine. The mass flow rates of the different injectors differ by no less than $3 \%$ and no more than $10 \%$. Therefore, as an approximation, the total mass flow rate was divided by the total number of injectors (271) and applied to this single injector configuration.

\section{Experimental Apparatus}

A test apparatus was designed based on the scaling criteria and the full scale conditions of the RD-170 engine. The piping schematic of the designed apparatus can be seen in Fig. 4. The propellants chosen were oxygen, methane and propane with nitrogen as the diluent for both the fuel and oxidizer. Methane and propane were selected due to the differing molecular weights that enabled a wider range of operating conditions to be realized. 
The propellants are supplied to the feed system by standard pressurized k-bottles, except the propane which is stored as a liquid at atmospheric temperature. Each high pressure gas is decompressed with a dual-stage regulator, which is set to allow sufficient mass flow for testing. Appropriately sized digital mass flow controllers were selected to regulate the flow of each propellant or diluent. The digital capability allows for an automated control system incorporated into the data acquisition system. Heat is added to each gas through an in-line heater. The power to each heater is controlled by an independent phase angle firing system to ensure precise control of each gas temperature. Once the gases are heated to the correct temperature they are mixed in a collector. The collectors were designed to mix the propellant constituents into a homogeneous mixture. Both the fuel and oxidizer collector contain a baffle compartment to minimize chamber oscillations from penetrating into the feed line and enhance mixing. Downstream of the collectors, a pressure reading is taken at the injector manifold inlet to determine the pressure drop across the injector. The injector manifold holds a single full scale injector and was designed to be compatible with multiple injector geometries and mixing schemes. To monitor and control the gaseous propellants, seven temperature readings are taken in the feed system by type $T$ thermocouples. To ensure safe operation, appropriate safety considerations were designed into the test facility. Check valves are applied to the system to prevent backflow. The nitrogen used to dilute the propellants serves a second purpose in the test facility. When beginning a test, nitrogen flows through each of the feed lines to avoid overheating the heater elements, and approach a gas temperature near testing conditions. After testing, the nitrogen system is activated and the propellants are shut off to purge the system of any unburned reactive gases.

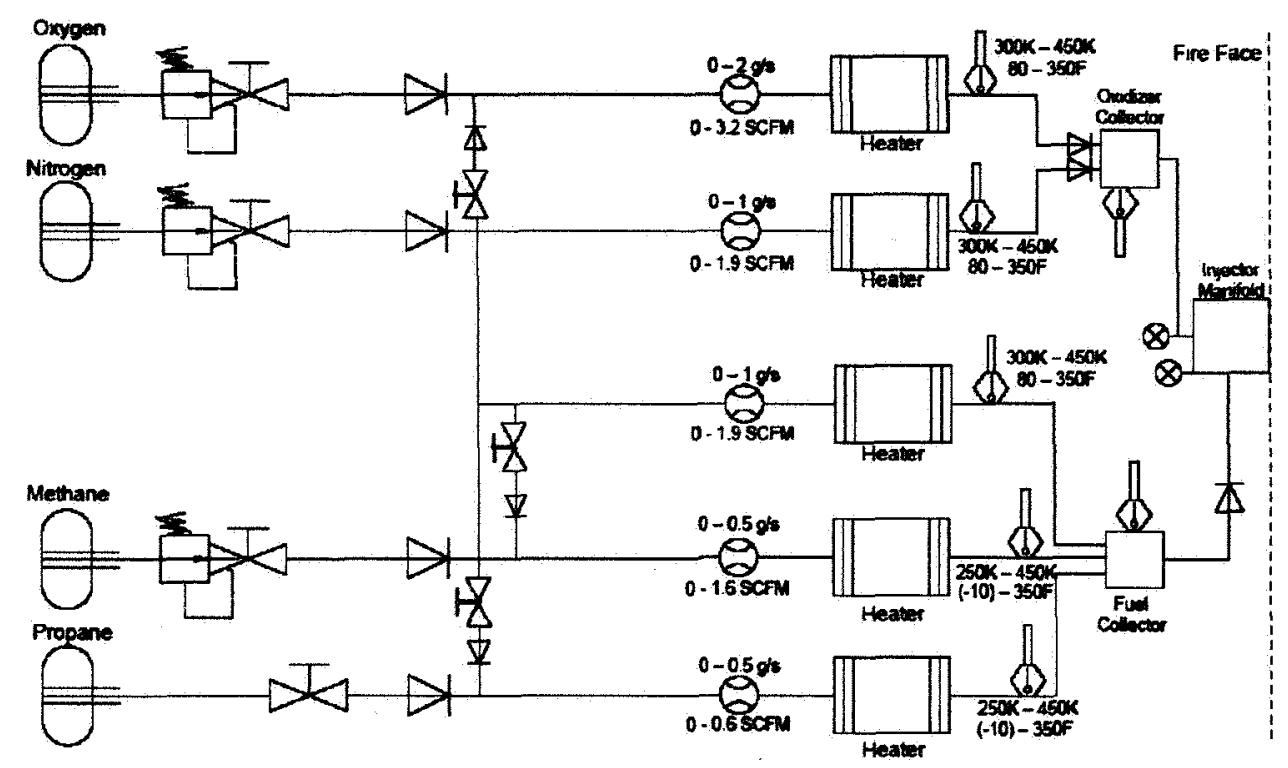

Figure 4. UAH Test Facility Schematic

A LabVIEW virtual instrument has been developed with a separate experimental apparatus testing high frequency combustion instability. The program incorporates a high frequency pressure input which goes through a Fast Fourier Transform spectrum analysis that is displayed and recorded in real time. The program also controls testing procedures. An analog signal is a control output for apparatus component management. This code is being modified to control the new equipment. Algorithms are being developed to control temperature, mass flow rate, and the testing sequence with the nitrogen purge system beginning and finishing the test. The program will simultaneously monitor and display chamber pressure oscillations during firing.

\section{Test Matrix Design Results}

Many dimensionless criteria were considered for testing. Conventional fluid dynamic parameters, Reynolds number, Mach number, and Euler number were calculated for possible full scale and subscale matching. The researchers at KARI applied a different scaling parameter (II) that was considered as well. The following list describes the dimensionless parameters in the forms used for subsequent calculations. 
- Reynolds Number

$$
\operatorname{Re}_{D, a x}=\frac{\rho_{o x} W_{o x} D_{a x}}{\mu_{a x}} \quad \operatorname{Re}_{D, F}=\frac{\rho_{F} W_{\Sigma} D_{F}}{\mu_{F}}
$$

In the case of the oxidizer the characteristic diameter $\left(D_{o x}\right)$ is the oxygen post diameter, and the velocity $\left(W_{o x}\right)$ is determined based on compressible flow theory for jet injector design. ${ }^{8}$ The fuel parameters are based on swirl injector design calculations. The characteristic diameter $\left(D_{F}\right)$ is the injector exit nozzle diameter. The discharge velocity $\left(\mathrm{W}_{\Sigma}\right)$, which is the magnitude of the vector sum of the axial, radial and tangential velocity components, is taken as the velocity.

- Mach Number

$$
M a_{o x}=\frac{W_{o x}}{c_{a x}} \quad M a_{F}=\frac{W_{\Sigma}}{c_{F}}
$$

The Mach number for the oxidizer is straight forward, applying the axial velocity and the speed of the sound $\left(c_{o x}\right)$ of the oxidizer in the oxygen post. The fuel Mach number is based on the discharge velocity and the speed of sound $\left(c_{F}\right)$ of the fuel at the injector manifold inlet temperature.

- Euler Number

$$
E u_{o x}=\frac{\Delta P_{o x} D_{o x}}{1 / 2 \rho_{o x} W_{o x}^{2} L_{o x}} \quad E u_{F}=\frac{\Delta P_{F} D_{F}}{1 / 2 \rho_{F} W_{\Sigma}^{2} L_{F}}
$$

The conventional Euler number has been modified to incorporate the characteristic diameter to length ratio. The oxidizer applies the parameters previously described as well as a characteristic length $\left(L_{o x}\right)$, which was taken as the length of the oxidizer post. The characteristic length $\left(\mathrm{L}_{\mathrm{F}}\right)$ of the fuel section is the sum of the mixing zone and tangential recess. For the RD-170 injector this length is $18.5 \mathrm{~mm}$.

- PI Criterion

$$
\Pi=\frac{W_{a x}}{W_{F}} \sqrt{\frac{\rho_{a x}}{\rho_{F}}}=\frac{A_{F}}{A_{a x}} \frac{n \&_{b x}}{n \&_{F}} \sqrt{\frac{\rho_{F}}{\rho_{a x}}}=k \frac{n \&_{b x}}{n \&_{F}} \sqrt{\frac{\rho_{F}}{\rho_{o x}}}=\frac{n \&_{a x}}{n \&_{F}} \sqrt{\frac{\rho_{F}}{\rho_{o x}}}
$$

The PI dimensionless criterion is the square root of the ratio of the kinetic energies which has proven to be significant for stability characterization. Lee et $\mathrm{al}^{5}$. have simplified the equation for an experimentally usable format as seen in Eq. 4. The simplification exposes an area ratio (k) that has been dropped due to the fact that the injector geometry will be constant for all tests. In a design application where injector geometries change, the area ratio would be necessary for comparison of injectors with differing geometries.

\section{A. Test Apparatus Capabilities}

The previously defined parameters were exercised to find the potential operating ranges of the designed test facility. Table 2 lists the results of parametric studies with the RD-170 injector. The calculation results are based on the temperature ranges and mass flow rates specified in Fig. 4. Significant assumptions had to be applied for the subscale operating range calculations. The mass flow controllers were assumed to throttle 10:1. Also, the mass fraction of the inert gas did not exceed $33 \%$ to avoid flammability limits that could be encountered while testing with substantial inert proportions. Another concern with significant quantities of diluent is the similarity of 
propellants with the added neutral gas. The pressure drop across the injector is used directly in the Euler number as well as being a parameter to determine the discharge velocity of the swirling fuel and the axial velocity of the oxidizer. It is customary to design the injector pressure drop at $10 \%$ of the chamber pressure to avoid chugging instabilities. Indeed, this is the case for the full scale RD-170 engine. Without experimental data to map the flow rate dependence on the pressure drop, a constant value of $10.3 \mathrm{kPa}, 10 \%$ of atmospheric pressure, was applied.

Table 2. Full Scale Parameters and Subscale Parameter Ranges

\begin{tabular}{|c|c|c|c|c|}
\hline & \multicolumn{2}{|c|}{ 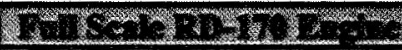 } & \multicolumn{2}{|c|}{ 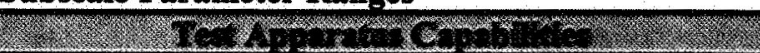 } \\
\hline Thandor & RP-1 & Oxygen & Fuel & Oxidizer \\
\hline Reynolds Number & $2.76 \mathrm{E}+06$ & $8.19 \mathrm{E}+06$ & $7.65 \mathrm{E}+04$ to $4.19 \mathrm{E}+05$ & $7.86 \mathrm{E}+02$ to $5.25 \mathrm{E}+04$ \\
\hline Euler Number & 0.757 & 0.132 & 0.757 & 0.145 \\
\hline Mach Number & 0.087 & 0.337 & 0.387 to 0.429 & 0.367 to 0.370 \\
\hline PI Criterion & \multicolumn{2}{|c|}{6.355} & \multicolumn{2}{|c|}{0.096 to 96.716} \\
\hline Volumetric Flow Rate $\left[\mathrm{m}^{3} / \mathrm{s}\right]$ & $7.23 \mathrm{E}-04$ & $1.05 \mathrm{E}-02$ & $2.33 \mathrm{E}-05$ to $2.29 \mathrm{E}-3$ & $1.54 \mathrm{E}-04$ to $3.63 \mathrm{E}-03$ \\
\hline Mixture Ratio & \multicolumn{2}{|c|}{2.6} & \multicolumn{2}{|c|}{0.1 to 40.0} \\
\hline
\end{tabular}

\section{B. Chamber Sizing}

To correctly scale the combustion chamber, the natural frequency of the tangential mode must be the same for the full scale and subscale chambers. This frequency is a function of the chamber diameter and chamber speed of sound.

$$
D_{m}=D_{f s} \frac{c_{m}}{c_{f s}}
$$

The full scale RD-170 main combustion chamber diameter is $380 \mathrm{~mm}$. Analysis of the combustion products was required to determine the speed of sound in the full scale chamber. Drawing on the aforementioned full scale operation conditions, the product composition was determined using GASEQ. RP-1 was not available as a reactant, so decane was used as an approximation. To check this approximation the temperature of the simulated combustion was compared to the value specified in Reference 6. The temperature comparison supported the use of decane as a fuel. The speed of sound in the full scale engine was calculated as $1326 \mathrm{~m} / \mathrm{s}$. Parametric studies determined an average speed of sound for representative subscale operating conditions. The first set of conditions was with methane at various mixture ratios, and the same conditions with maximum nitrogen dilution. A similar analysis was used with propane as the fuel. The results did not change significantly for the evaluated cases. The average speed of sound for all conditions was $1220 \mathrm{~m} / \mathrm{s}$. Eq. (5) was implemented to determine the diameter of the model combustion chamber at $350 \mathrm{~mm}$.

\section{Discussion}

This scaling methodology follows the Russian scaling tradition, which is to extract a section of the full scale device and closely match the boundary conditions. Unfortunately, matching the full scale boundary conditions can be extremely difficult. For instance, the conception of phase matching is presented but there is no detailed direction for the test apparatus design. In this case, the processes of the full scale engine have been described but without explicitly explaining how to achieve similarity. With a limited knowledge of the full scale manifold, the phase of the injection process may be assumed as a secondary effect for the initial apparatus design. Further research will be required to incorporate phase matching.

The proclamation that the mean chamber pressure exerts no principle influence on the chamber acoustic field spatial parameters must be further scrutinized. The difference between the energy entering the chamber and the energy leaving the chamber is the acoustic energy in the chamber. A near atmospheric chamber will not achieve sonic flow through the nozzle. Therefore, the losses exiting the model chamber nozzle may not be similar to the losses exiting the full scale engine nozzle. In this case, proper considerations must be made to ensure the entering energy is scaled correctly. The chamber could be placed in a vacuum to avoid this inconsistency.

Table 2 indicates that the experimental apparatus can achieve wide ranges of parameters. Unfortunately for this injector, many of the dimensionless parameters do not approach the full scale conditions. With such low mass flow 
rates and thermodynamic restrictions, the maximum model Reynolds number is low by orders of magnitude compared to the full scale. The researchers hope was to approach a regime of auto-modality and assume similar turbulence conditions in the model and full scale engine. The Mach numbers are on the correct order of magnitude. Apparatus modifications can be made to change the gaseous temperature which will have the greatest affect on the Mach number. The Euler number is only a rough approximation without testing experience.

Once an experimental pressure drop study is performed, a realistic view of testing capabilities will be obtained. The pressure drop approximation affects the jet portion of the injector as well. The jet oxidizer velocity and the dimensionless parameters dependent on velocity will also be corrected with pressure drop data. The initial literature review did not expose a method to calculate the parameters of a gaseous open nozzle swirl injector. As a first approximation, the equations applied to the model swirling fuel were taken on the assumption that the propellants were liquid. For the full scale injector this is accurate, but for the model conditions the fuel is a compressible gas.

The pertinent parameters of the study, volumetric flow rate and the PI criterion are independent of the pressure drop assumption. The volumetric flow rate is the basic requirement for this scaling technique. The test apparatus successfully matches the fuel volumetric flow rate. In fact, the range suggested by KARI researchers is exceeded. The apparatus oxidizer volumetric flow rate capability does not cover the necessary range. The oxidizer volumetric flow rate may be increased by a greater mass fraction of nitrogen. In the case of matching the fuel criteria without nitrogen, a greater mass fraction of oxidizer inert gas can be rationalized. The most promising scaling factor is the PI criterion. This dimensionless parameter may be most useful for multiple reasons. First, it is the only parameter used by Lee et al. besides volumetric flow rate matching. Secondly, the development of this Russian technique was at the NIICHIMMASH design bureau. KARI was able to reference reports directly from these originators, so there may be a greater reason for this parameter. The PI criterion is successfully scaled with the designed test apparatus. In fact, the logarithm of the full scale value is near the center of the logarithmic operating range, which will allow more flexibility while matching the volumetric flow rate.

The greatest source of error comes from the method of chamber scaling. Assuming that the temperature of the products in the full scale engine is the uniform temperature of the chamber volume is a good approximation. However, for the model chamber multiple problems are encountered. The operating conditions substantially differ with varying fuels and inert gas mass fractions. The flame temperature will vary under all of these conditions. The greater problem is that the chamber will experience a significant temperature gradient due to the placement of the single injector near the chamber wall. The temperature will be the dominating factor defining the speed of sound in this test facility. Experimentally determining the chamber temperature would be an improved technique for chamber sizing. In fact, the researchers at KARI experimentally determined the temperature in the model chamber. In this analytical approach, the results for the model chamber are near to the original size of the full scale engine. Therefore multiple chamber sizes have been designed, accounting for the cold gas region in the model chamber.

\section{Recommendations}

The secondary objective of this work is to improve the experimental technique. In order to improve the methodology, testing will be required. Through the design process, multiple improvements have been speculated. The suggested chamber sizing process seems insufficient for this single element test. A variable size chamber would allow a more simplistic approach to a test apparatus design. No assumptions would be required about the model chamber conditions. The variable width design also alleviates the need for fabricating multiple chambers when testing several injectors under various conditions. The variable width chamber would be rectangular in cross section with three fixed walls and a smoothly variable wall. This rectangular geometry would be compatible with the low pressure oscillation amplitude. The chamber could be tuned with the variable wall to match the full scale tangential frequency while testing. This chamber has an added advantage for simpler optical access through the flat walls. A high speed camera or chemiluminescence measurements would enhance the data collection to better understand the combustion phenomena.

The second improvement would be to instrument the apparatus with a multiplicity of high frequency pressure transducers. In accordance with NASA SP-194 ${ }^{9}$, multiple high pressure transducers will be placed in the chamber to distinguish spinning modes or combined modes of combustion instability. An added advantage beyond the designed apparatus would be gained from mounting transducers in the propellant manifolds.

The application of monopropellant injectors is an attractive idea. With the appropriate arrangement, a cluster of monopropellant injectors may be applicable. A portion of the fire face could be taken with monopropellant swirl injectors or impinging jets. Many configurations would be necessary to find a representative cluster that would demonstrate similarity. This scaling approach would be a subject of future research to determine accuracy. 


\section{Acknowledgments}

This work is sponsored by NASA under Contract NNM05AA22A. The authors would like to thank Tom Nesman and Jim Hulka of Marshall Space Flight Center and Jacobs Sverdrup, respectively for their technical advice and direction, also to Dr. Marlow Moser of the UAH Propulsion Research Center for input on the test facility design.

\section{References}

${ }^{1}$ Fisher, S. C., Dodd, F. E., and Jensen, R. J., "Scaling Techniques for Liquid Rocket Combustion Stability Testing," Progress in Astronautics and Aeronautics, Vol. 169, 1995. Liquid Rocket Engine Combustion Instability, ed. V. Yang, and W. E. Anderson, AIAA.

${ }^{2}$ Penner, S. S., "On the Development of Rational Scaling Procedures for Liquid-Fuel Rocket Engines," Jet Propulsion, Feb. 1957, pp.156-68.

${ }^{3}$ Penner, S. S., and Fuhs, A. E., "On Generalized Scaling Procedures for Liquid-Fuel Rocket Engines," Combustion and Flame, Vol. 1, 1957, pp. 229-240.

${ }^{4}$ Dexter, C.E, Hulka, J. et al., "Scaling Techniques for Design, Development, and Test", Progress in Astronautics and Aeronautics Series, Vol. 200, 2004. Liquid Rocket Thrust Chamber: Aspects of Modeling, Analysis, and Design. ed.Yang, V., Habiballah, M. et al., AIAA.

${ }^{5}$ Lee, K., Seo, S., Song, J., Han, Y., Choi, H., Seol, W., "Combustion Stability Assessment of Double Swirl Coaxial Injectors Using Simulant Propellants," AIAA Paper 2005-4443, July 2005.

${ }^{6}$ Manski, D., et al., "Cycles for Earth-to-Orbit Propulsion," Journal of Propulsion and Power, Vol. 14, No. 5, 1998, pp. 588604.

${ }^{7}$ Vasin, A., et al., Otkrytoe Aktsioneroe Obschestvo "Nauchao-Proizvodatveabnoe Obiedianie", U.S. Patent Application for a "Liquid-propellant rocket engine chamber and its casing," 6,244,041, filed 10 Sept. 1999.

${ }^{8}$ Bazarov, V., Yang, V., Puri, P., "Chapter 2: Design and Dynamics of Jet and Swirl Injectors," in Progress in Astronautics and Aeronautics Series, Vol. 200, 2004. Liquid Rocket Thrust Chamber: Aspects of Modeling, Analysis, and Design. ed.Yang, V., Habiballah, M. et al., AIAA.

${ }^{9}$ Harrje, D. T., Reardon, F. H., "Liquid Propellant Rocket Combustion Instability", NASA SP-194, 1972.

${ }^{10}$ Bazarov, V. G., Liquid Injector Dynamics, Mashinostroenie, Moscow, 1979. 


\title{
Experimental Methodology for Measuring Combustion and Injection-Coupled Responses
}

\author{
Ryan C. Cavitt", Robert A. Frederick, Jr. ${ }^{\dagger}$, and Vladimir G. Bazarov ${ }^{\ddagger}$ \\ UAH Propulsion Research Center, Huntsville, Alabama, 35899
}

\begin{abstract}
A Russian scaling methodology for liquid rocket engines utilizing a single, full scale element is reviewed. The scaling methodology exploits the supercritical phase of the full scale propellants to simplify scaling requirements. Many assumptions are utilized in the derivation of the scaling criteria. A test apparatus design is presented to implement the Russian methodology and consequently verify the assumptions. This test apparatus will allow researchers to assess the usefulness of the scaling procedures and possibly enhance the methodology. A matrix of the apparatus capabilities for a RD-170 injector is also presented. Several methods to enhance the methodology have been generated through the design process.
\end{abstract}

$\begin{array}{ll}\mathbf{c} & =\text { Speed of sound } \\ \mathbf{D} & =\text { Diameter } \\ \mathbf{E u} & =\text { Euler Number } \\ \mathbf{k} & =\text { Area ratio } \\ \mathbf{L} & =\text { Length } \\ n \boldsymbol{n} & =\text { Mass flow rate } \\ \mathbf{M a} & =\text { Mach Number } \\ \Delta \mathbf{P} & =\text { Pressure drop across injector } \\ \mathbf{R e} & =\text { Reynolds Number } \\ \mathbf{\rho} & =\text { Density } \\ \mathbf{W} & =\text { Velocity }\end{array}$

Nomenclature

$$
\begin{aligned}
& \text { Subscripts } \\
& F=\text { fuel } \\
& \mathbf{f}=\text { full scale } \\
& \mathbf{m}=\text { model } \\
& \mathbf{o x}=\text { oxidizer } \\
& \mathbf{\Sigma}=\text { discharge }
\end{aligned}
$$

\section{Introduction}

$\mathrm{H}$ igh frequency combustion instability continues to hinder the development of liquid rocket engines (LRE). Unstable combustion is characterized by high amplitude chamber pressure fluctuations at distinct frequencies. In extreme cases, chamber pressure oscillations can lead to catastrophic loss of hardware. This destructive phenomenon can appear and destroy an engine in fractions of a second. ${ }^{1}$ Combustion instability is an interaction between the natural frequency of the engine hardware and the combustion process. Therefore, propellant injection is a critical component in the system. The complex processes of mixing, atomization and vaporization are proclaimed to be the leading processes responsible for instability.

Designing a LRE to circumvent this problem has proven to be a formidable task. The most straightforward approach to determine the stability of a LRE is to test the full scale engine. Unfortunately, this is usually not a feasible or efficient approach. Full scale testing is extremely expensive and time consuming. If full scale testing is to be used solely, the LRE designer is forced to rely completely on previous designs and experience for the initial design. In the event of unstable operation, modifications are required. Passive suppression methods such as baffles and resonators are commonly employed, but can prove insufficient. In this case, more extreme changes can be necessary at the cost of excessive time and resources. Engine stability considerations must be implemented early in

\footnotetext{
* Graduate Research Assistant, UAH Propulsion Research Center, 5000 Technology Drive, and AIAA Member.

${ }^{\dagger}$ Assistant Director, UAH Propulsion Research Center, 5000 Technology Drive, and AIAA Associate Fellow.

‡ Visiting Eminent Scholar of Propulsion, UAH Propulsion Research Center, 5000 Technology Drive, and AIAA Member.
} 
the design process to avoid modifying designed hardware to obtain stable combustion. Subscale testing can be implemented to avoid such a problematic cycle. A successful subscale experimental program will never substitute for full scale testing, but can aid in reducing the chances of experiencing high frequency combustion instability in the final design. Scaling can be the solution to stability characterization. Scaling procedures were heavily developed in the 1950's. ${ }^{2,3}$ Many scaling techniques are based on the dimensionless analysis of fluid dynamic and combustion criteria such as Reynolds number, Prandtl number and Damkb̈hler number. To derive the scaling procedures, assumptions and limitations must be invoked. These assumptions and limitations make certain techniques incompatible with unique engines. Achieving similarity can be difficult or even impossible with an appropriate methodology in practice.

Many different approaches to subscale testing exist. Single element injector testing is obviously the simplest form of scaling. The use of only one injector limits the information that can be extracted from experiments. Intraelement phenomena are the only characteristics that can be represented by the single element technique. Injector flow and combustion characteristics are of key importance and can be taken from such testing. In the event of stable combustion at nominal operating conditions, stability rating can be used by varying operating conditions to identify the boundaries of instability and subsequently how far the operating conditions are from unstable operation. ${ }^{4}$ For instance, fuel mass flow rate can be throttled, changing the mixture ratio, to isolate feed system dynamic affects, also chamber pressure variations aid in scrutinizing atomization and vaporization affect.

With any scaling procedure there are limitations. The single injector method neglects interelement influences. The influence of neighboring sprays can have a significant affect on the overall stability of the system, which must be investigated with other means. Similarity matching are of utmost importance not only for the injector, but also the combustion chamber conditions. Scaling the full scale chamber conditions is not simple. Assumptions about the chamber conditions must be applied. The uniform temperature of the chamber is customarily taken as the temperature of the combustion products. This may not be an accurate assumption. The most dangerous modes of instability, from a catastrophic failure standpoint, are the tangential modes. In this instance, the maximum pressure fluctuations are occurring at the injector fire face where the temperature can be influenced by heat loss through the fire face and recirculation flow creating a complex temperature field. If the product temperature is used to determine the speed of sound of the full scale combustion chamber, an inaccurate condition is supplied to the model.

\section{Background}

The recent publication by Dexter, et al. ${ }^{4}$, has described a Russian methodology that remains to be tested in the U.S. The methodology was developed during the design of the RD-170 engine, which ultimately utilized a gasliquid injector. This technique takes advantage of the supercritical state of the propellants prior to injection in the full scale engine. A supercritical fluid is identified as a dense gas; therefore surface tension does not exist. This is the greatest simplification to enable successful scaling. The absence of surface tension indicates that the atomization and vaporization processes are not important for scaling. Modeling can be achieved without matching droplet similarity conditions such as Weber number and Ohnesorge number. This leaves mixing as the leading process in high frequency combustion instability to be scrutinized.

\section{A. Experimental Setup}

A schematic of the methodology experimental device can be seen in Fig. 1. A near atmospheric pressure, water cooled chamber is used to allow long duration firings. The chamber operates near atmospheric pressure following the proclamation that the mean chamber pressure has minimal influence on the chamber acoustic field spatial parameters. One of the advantages of the atmospheric pressure chamber is that the hardware can function and endure the pressure oscillation amplitude that will be excited for the duration of the test. A single full scale, bipropellant injector is placed near the chamber wall to excite tangential modes of combustion instability. For full scale engines with multiple injector designs residing in a single chamber, a representative cluster of bipropellant injectors can be substituted for the single injector. The injector or injector cluster is fired with gaseous propellants, which is a consequence of full scale supercritical propellants. The oxidizer is heated to change the thermodynamic properties entering the injector. A neutral gas can be introduced to aid in matching the discharge velocity of the oxidizer. In addition, the neutral gas will influence the thermodynamic properties of the mixture. A similar feed system is used for the fuel, with the absence of a heating element. The minimum data acquisition requirement is a high frequency pressure transducer monitoring pressure fluctuations in the combustion chamber.

The methodology publication lists many other scaling criteria. First, partial modeling must be applied. Choosing the correct similarity conditions is crucial to accurately represent the processes in the full scale engine. Second, 
sizing the chamber is required to match the transverse frequency. This includes the chamber dimensions and speeds of sound of the model and full scale engine. Lastly, two phases must be matched; the chamber acoustics and combustion process, as well as the manifold acoustics and injection process. The first involves the chamber acoustics, which have been addressed and the time delay for the reactants to convert to products. The second involves scaling the injector manifold geometry and propellant temperature to match the time for an acoustic disturbance to traverse the manifold.

The Korean Aerospace Research Institute (KARI) has implemented this Russian methodology to investigate recess ratio effects in double swirl injectors. ${ }^{5}$ The described test setup was assembled with a cluster of five injectors instead of a single injector. A scaling parameter (I) was developed to meet suite testing procedures and was implemented for the partial modeling criteria. The results of the study agree with common injector trends. As the recess ratio increases, mixing increases which increases combustion efficiency and instability. The successful use of this methodology beyond the original jet-swirl application is promising for use in other injector schemes.

\section{B. Testing Procedure}

An experimental procedure is suggested with this methodology description. A graphic representation can be seen in Fig. 2. The graph illustrates the following testing procedure for a single injector. The fuel mass flow rate is fixed and the oxidizer mass flow rate is smoothly varied to a maximum value and returned to the minimum. The chamber pressure is recorded continuously throughout this experiment. A new fuel mass flow rate is selected and the procedure is repeated until sufficient data is acquired surrounding the design point operating conditions. In order to use the testing procedure effectively, the scaled down design point operating conditions from the full scale engine must be determined prior to the creation of the test matrix. Lee, et al. ${ }^{5}$ suggest varying the oxidizer volumetric flow rate from one half to twice the oxidizer volumetric flow rate design point. To define the threshold between stable and unstable combustion, a pressure oscillation level must be set. Data analysis of the pressure fluctuations with a set limit of instability will define the boundary of instability at different operating conditions.

When utilizing this experiment during engine development, mapping the boundaries of instability can drive the design of injectors toward the stable regions. Ideally, the final design point would lie far from the instability region. This distance can be quantified via stability margin rating and used to compare different injector designs. Once a model injector design and operating conditions are finalized, the test injector is equivalent to the full scale injector and the subscale operating conditions can be extrapolated to the full scale conditions.

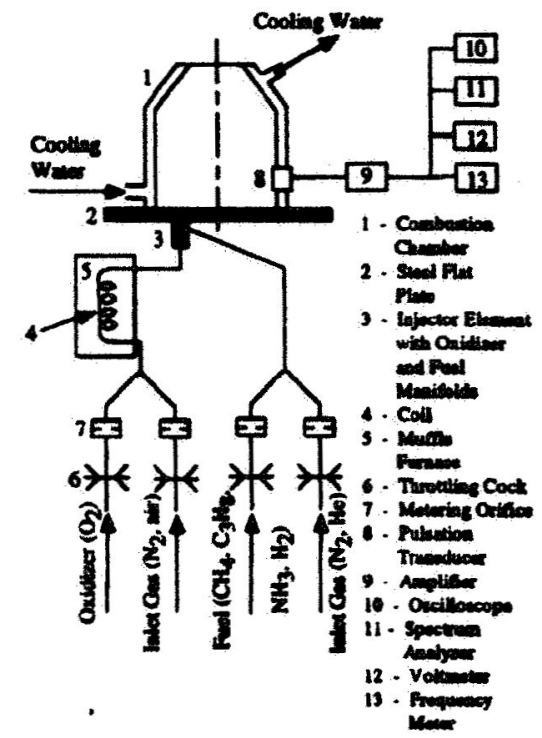

Figure 1. Russian Methodology Test Apparatus Schematic ${ }^{4}$

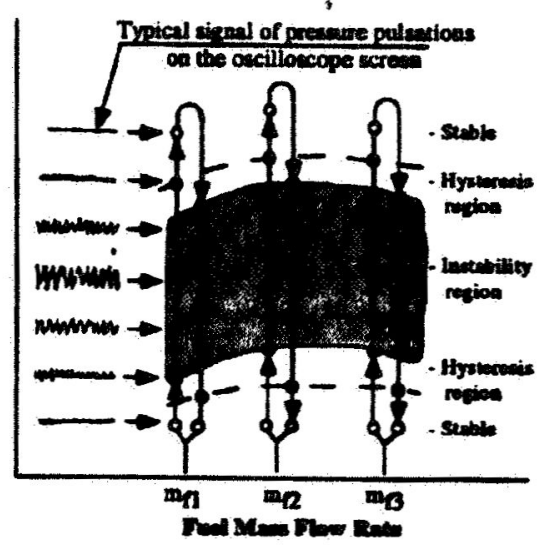

Figure 2. Boundary of Instability Testing Procedure ${ }^{4}$

\section{Approach}

\section{A. Objective}

The main objective of this project is to determine the feasibility of this simplistic Russian scaling technique. Determining the accuracy of the methodology could give LRE designers an invaluable tool to utilize throughout the engine development process. By verifying the scaling technique, strict adherence to the procedures can allow LRE designers to test multiple injector schemes and geometries and scale the test conditions up to full scale conditions for the final design. The assessment includes a scaling literature review, test apparatus design and experiments to verify the assumptions of the scaling procedures. Three injector schemes were selected for characterization; impinging jet, jet-swirl and swirl-swirl. A secondary objective of the project is to enhance the methodology, if possible 
improvements become evident. The inefficiencies or shortfalls of the scaling procedure will be exposed through the design process and experimental results.

\section{B. Injector Operating Conditions}

Since the methodology was created and implemented during the development of the RD-170 engine, the first injector to be tested is the injector from the main combustion chamber of this engine. Therefore the first set of tests will be to replicate the testing undergone by the Russian designers. The injector is a jet-swirl configuration with oxygen supplied axially and RP-1 introduced tangentially. The injector can be seen in Fig. 3.

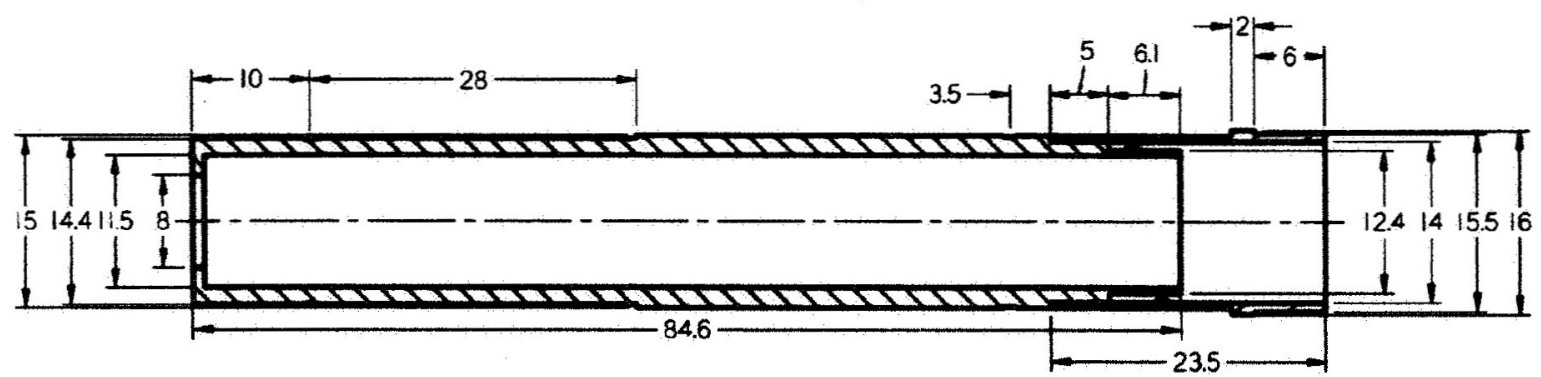

Figure 3. RD-170 Main Combustion Chamber Injector (all dimensions in mm)

The pertinent overall operating conditions for the RD-170 engine were determined by Manski, et al. ${ }^{6} \mathrm{~A}$ summary of the propellant conditions directly upstream of the injector manifold can be seen in Table 1 . These values were used to determine the full scale dimensionless parameters.

Table 1. RD-170 Main Combustion Chamber Operating Conditions

\begin{tabular}{|c|c|c|}
\hline Trestate & $\Gamma$ & 0 \\
\hline Critical Temperature [K] & 676 & 154.6 \\
\hline Critical Pressure [bar] & 21.7 & 50.4 \\
\hline Density [kg/m^3] & 807 & 155.38 \\
\hline Pressure [bar] & 278.26 & 278.26 \\
\hline Temperature [K] & 492.2 & 687.7 \\
\hline Total Mass Flow Rate [kg/s] & 158.05 & 440.7 \\
\hline Injector Average Mass Flow Rate [kg/s] & 0.583 & 1.626 \\
\hline Pressure Drop [bar] & 25.3 & 25.3 \\
\hline Viscosity [kg/m-s] & $3.237 \mathrm{E}-04$ & $4.001 \mathrm{E}-05$ \\
\hline Discharge Velocity [m/s] & 79.18 & 183.40 \\
\hline Speed of Sound [m/s] & 907.9 & 543.5 \\
\hline
\end{tabular}

Examination of Table 1 will reveal that the RP-1 is not above the critical temperature and therefore does not satisfy the supercritical phase criterion. The propellant is below the critical temperature by $183 \mathrm{~K}$. An assumption about the injector thermal environment and combustion chamber must be made to allow the methodology to be used in this particular engine. As the fuel swirls around the nozzle, the RP-1 will be heated through the interaction with the heated oxygen spray. Also, the time for the fuel to vaporize in the combustion chamber is assumed to be negligible compared to the time for mixing.

The main combustion chamber of the RD-170 engine uses multiple injector types. ${ }^{7}$ The U.S. patent application of this engine was referenced to verify the use of the overall operating conditions to a single injector. At least five different injector designs are employed in the engine. The mass flow rates of the different injectors differ by no less than $3 \%$ and no more than $10 \%$. Therefore, as an approximation, the total mass flow rate was divided by the total number of injectors (271) and applied to this single injector configuration.

\section{Experimental Apparatus}

A test apparatus was designed based on the scaling criteria and the full scale conditions of the RD-170 engine. The piping schematic of the designed apparatus can be seen in Fig. 4. The propellants chosen were oxygen, methane and propane with nitrogen as the diluent for both the fuel and oxidizer. Methane and propane were selected due to the differing molecular weights that enabled a wider range of operating conditions to be realized. 
The propellants are supplied to the feed system by standard pressurized k-bottles, except the propane which is stored as a liquid at atmospheric temperature. Each high pressure gas is decompressed with a dual-stage regulator, which is set to allow sufficient mass flow for testing. Appropriately sized digital mass flow controllers were selected to regulate the flow of each propellant or diluent. The digital capability allows for an automated control system incorporated into the data acquisition system. Heat is added to each gas through an in-line heater. The power to each heater is controlled by an independent phase angle firing system to ensure precise control of each gas temperature. Once the gases are heated to the correct temperature they are mixed in a collector. The collectors were designed to mix the propellant constituents into a homogeneous mixture. Both the fuel and oxidizer collector contain a baffle compartment to minimize chamber oscillations from penetrating into the feed line and enhance mixing. Downstream of the collectors, a pressure reading is taken at the injector manifold inlet to determine the pressure drop across the injector. The injector manifold holds a single full scale injector and was designed to be compatible with multiple injector geometries and mixing schemes. To monitor and control the gaseous propellants, seven temperature readings are taken in the feed system by type $T$ thermocouples. To ensure safe operation, appropriate safety considerations were designed into the test facility. Check valves are applied to the system to prevent backflow. The nitrogen used to dilute the propellants serves a second purpose in the test facility. When beginning a test, nitrogen flows through each of the feed lines to avoid overheating the heater elements, and approach a gas temperature near testing conditions. After testing, the nitrogen system is activated and the propellants are shut off to purge the system of any unburned reactive gases.

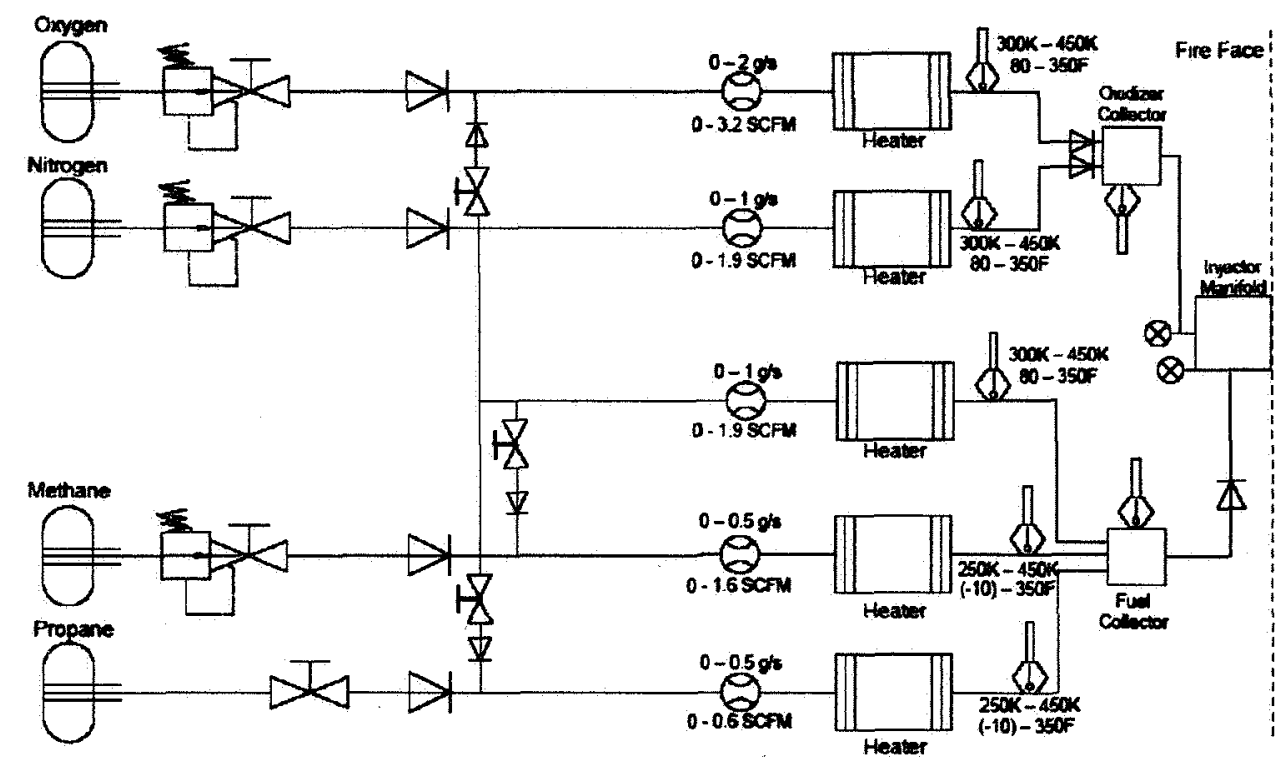

Figure 4. UAH Test Facility Schematic

A LabVIEW virtual instrument has been developed with a separate experimental apparatus testing high frequency combustion instability. The program incorporates a high frequency pressure input which goes through a Fast Fourier Transform spectrum analysis that is displayed and recorded in real time. The program also controls testing procedures. An analog signal is a control output for apparatus component management. This code is being modified to control the new equipment. Algorithms are being developed to control temperature, mass flow rate, and the testing sequence with the nitrogen purge system beginning and finishing the test. The program will simultaneously monitor and display chamber pressure oscillations during firing.

\section{Test Matrix Design Results}

Many dimensionless criteria were considered for testing. Conventional fluid dynamic parameters, Reynolds number, Mach number, and Euler number were calculated for possible full scale and subscale matching. The researchers at KARI applied a different scaling parameter (II) that was considered as well. The following list describes the dimensionless parameters in the forms used for subsequent calculations. 
- Reynolds Number

$$
\operatorname{Re}_{D, o x}=\frac{\rho_{o x} W_{o x} D_{a x}}{\mu_{o x}} \quad \operatorname{Re}_{D, F}=\frac{\rho_{F} W_{\Sigma} D_{F}}{\mu_{F}}
$$

In the case of the oxidizer the characteristic diameter $\left(D_{0 x}\right)$ is the oxygen post diameter, and the velocity $\left(\mathrm{W}_{\mathrm{ox}}\right)$ is determined based on compressible flow theory for jet injector design. ${ }^{8}$ The fuel parameters are based on swirl injector design calculations. The characteristic diameter $\left(D_{F}\right)$ is the injector exit nozzle diameter. The discharge velocity $\left(\mathrm{W}_{\Sigma}\right)$, which is the magnitude of the vector sum of the axial, radial and tangential velocity components, is taken as the velocity.

- Mach Number

$$
M a_{o x}=\frac{W_{a x}}{c_{o x}} \quad M a_{F}=\frac{W_{\Sigma}}{c_{F}}
$$

The Mach number for the oxidizer is straight forward, applying the axial velocity and the speed of the sound $\left(c_{o x}\right)$ of the oxidizer in the oxygen post. The fuel Mach number is based on the discharge velocity and the speed of sound $\left(\mathrm{c}_{\mathrm{F}}\right)$ of the fuel at the injector manifold inlet temperature.

- Euler Number

$$
E u_{o x}=\frac{\Delta P_{o x} D_{o x}}{1 / 2 \rho_{a x} W_{o x}^{2} L_{o x}} \quad E u_{F}=\frac{\Delta P_{F} D_{F}}{1 / 2 \rho_{F} W_{\Sigma}^{2} L_{F}}
$$

The conventional Euler number has been modified to incorporate the characteristic diameter to length ratio. The oxidizer applies the parameters previously described as well as a characteristic length $\left(L_{o x}\right)$, which was taken as the length of the oxidizer post. The characteristic length $\left(L_{F}\right)$ of the fuel section is the sum of the mixing zone and tangential recess. For the RD-170 injector this length is $18.5 \mathrm{~mm}$.

- PI Criterion

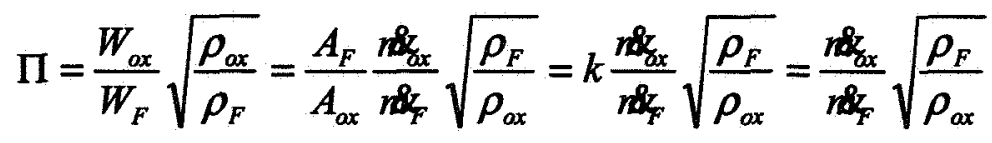

The PI dimensionless criterion is the square root of the ratio of the kinetic energies which has proven to be significant for stability characterization. Lee et $\mathrm{al}^{5}$. have simplified the equation for an experimentally usable format as seen in Eq. 4. The simplification exposes an area ratio (k) that has been dropped due to the fact that the injector geometry will be constant for all tests. In a design application where injector geometries change, the area ratio would be necessary for comparison of injectors with differing geometries.

\section{A. Test Apparatus Capabilities}

The previously defined parameters were exercised to find the potential operating ranges of the designed test facility. Table 2 lists the results of parametric studies with the RD-170 injector. The calculation results are based on the temperature ranges and mass flow rates specified in Fig. 4. Significant assumptions had to be applied for the subscale operating range calculations. The mass flow controllers were assumed to throttle 10:1. Also, the mass fraction of the inert gas did not exceed $33 \%$ to avoid flammability limits that could be encountered while testing with substantial inert proportions. Another concern with significant quantities of diluent is the similarity of 
propellants with the added neutral gas. The pressure drop across the injector is used directly in the Euler number as well as being a parameter to determine the discharge velocity of the swirling fuel and the axial velocity of the oxidizer. It is customary to design the injector pressure drop at $10 \%$ of the chamber pressure to avoid chugging instabilities. Indeed, this is the case for the full scale RD-170 engine. Without experimental data to map the flow rate dependence on the pressure drop, a constant value of $10.3 \mathrm{kPa}, 10 \%$ of atmospheric pressure, was applied.

Table 2. Full Scale Parameters and Subscale Parameter Ranges

\begin{tabular}{|c|c|c|c|c|}
\cline { 2 - 5 } \multicolumn{1}{c|}{} & RP-1 & Oxygen & Fuel & Oxidizer \\
\hline Reynolds Number & $2.76 \mathrm{E}+06$ & $8.19 \mathrm{E}+06$ & $7.65 \mathrm{E}+04$ to $4.19 \mathrm{E}+05$ & $7.86 \mathrm{E}+02$ to $5.25 \mathrm{E}+04$ \\
\hline Euler Number & 0.757 & 0.132 & 0.757 & 0.145 \\
\hline Mach Number & 0.087 & 0.337 & 0.387 to 0.429 & 0.367 to 0.370 \\
\hline PI Criterion & \multicolumn{3}{|c|}{6.355} & \multicolumn{2}{c|}{0.096 to 96.716} \\
\hline Volumetric Flow Rate [m $3 / \mathrm{s}]$ & $7.23 \mathrm{E}-04$ & $1.05 \mathrm{E}-02$ & $2.33 \mathrm{E}-05$ to $2.29 \mathrm{E}-3$ & $1.54 \mathrm{E}-04$ to $3.63 \mathrm{E}-03$ \\
\hline Mixture Ratio & \multicolumn{3}{|c|}{2.6} & \multicolumn{2}{c|}{0.1 to 40.0} \\
\hline
\end{tabular}

\section{B. Chamber Sizing}

To correctly scale the combustion chamber, the natural frequency of the tangential mode must be the same for the full scale and subscale chambers. This frequency is a function of the chamber diameter and chamber speed of sound.

$$
D_{m}=D_{f s} \frac{c_{m}}{c_{f s}}
$$

The full scale RD-170 main combustion chamber diameter is $380 \mathrm{~mm}$. Analysis of the combustion products was required to determine the speed of sound in the full scale chamber. Drawing on the aforementioned full scale operation conditions, the product composition was determined using GASEQ. RP-1 was not available as a reactant, so decane was used as an approximation. To check this approximation the temperature of the simulated combustion was compared to the value specified in Reference 6. The temperature comparison supported the use of decane as a fuel. The speed of sound in the full scale engine was calculated as $1326 \mathrm{~m} / \mathrm{s}$. Parametric studies determined an average speed of sound for representative subscale operating conditions. The first set of conditions was with methane at various mixture ratios, and the same conditions with maximum nitrogen dilution. A similar analysis was used with propane as the fuel. The results did not change significantly for the evaluated cases. The average speed of sound for all conditions was $1220 \mathrm{~m} / \mathrm{s}$. Eq. (5) was implemented to determine the diameter of the model combustion chamber at $350 \mathrm{~mm}$.

\section{Discussion}

This scaling methodology follows the Russian scaling tradition, which is to extract a section of the full scale device and closely match the boundary conditions. Unfortunately, matching the full scale boundary conditions can be extremely difficult. For instance, the conception of phase matching is presented but there is no detailed direction for the test apparatus design. In this case, the processes of the full scale engine have been described but without explicitly explaining how to achieve similarity. With a limited knowledge of the full scale manifold, the phase of the injection process may be assumed as a secondary effect for the initial apparatus design. Further research will be required to incorporate phase matching.

The proclamation that the mean chamber pressure exerts no principle influence on the chamber acoustic field spatial parameters must be further scrutinized. The difference between the energy entering the chamber and the energy leaving the chamber is the acoustic energy in the chamber. A near atmospheric chamber will not achieve sonic flow through the nozzle. Therefore, the losses exiting the model chamber nozzle may not be similar to the losses exiting the full scale engine nozzle. In this case, proper considerations must be made to ensure the entering energy is scaled correctly. The chamber could be placed in a vacuum to avoid this inconsistency.

Table 2 indicates that the experimental apparatus can achieve wide ranges of parameters. Unfortunately for this injector, many of the dimensionless parameters do not approach the full scale conditions. With such low mass flow 
rates and thermodynamic restrictions, the maximum model Reynolds number is low by orders of magnitude compared to the full scale. The researchers hope was to approach a regime of auto-modality and assume similar turbulence conditions in the model and full scale engine. The Mach numbers are on the correct order of magnitude. Apparatus modifications can be made to change the gaseous temperature which will have the greatest affect on the Mach number. The Euler number is only a rough approximation without testing experience.

Once an experimental pressure drop study is performed, a realistic view of testing capabilities will be obtained. The pressure drop approximation affects the jet portion of the injector as well. The jet oxidizer velocity and the dimensionless parameters dependent on velocity will also be corrected with pressure drop data. The initial literature review did not expose a method to calculate the parameters of a gaseous open nozzle swirl injector. As a first approximation, the equations applied to the model swirling fuel were taken on the assumption that the propellants were liquid. For the full scale injector this is accurate, but for the model conditions the fuel is a compressible gas.

The pertinent parameters of the study, volumetric flow rate and the PI criterion are independent of the pressure drop assumption. The volumetric flow rate is the basic requirement for this scaling technique. The test apparatus successfully matches the fuel volumetric flow rate. In fact, the range suggested by KARI researchers is exceeded. The apparatus oxidizer volumetric flow rate capability does not cover the necessary range. The oxidizer volumetric flow rate may be increased by a greater mass fraction of nitrogen. In the case of matching the fuel criteria without nitrogen, a greater mass fraction of oxidizer inert gas can be rationalized. The most promising scaling factor is the PI criterion. This dimensionless parameter may be most useful for multiple reasons. First, it is the only parameter used by Lee et al. besides volumetric flow rate matching. Secondly, the development of this Russian technique was at the NIICHIMMASH design bureau. KARI was able to reference reports directly from these originators, so there may be a greater reason for this parameter. The PI criterion is successfully scaled with the designed test apparatus. In fact, the logarithm of the full scale value is near the center of the logarithmic operating range, which will allow more flexibility while matching the volumetric flow rate.

The greatest source of error comes from the method of chamber scaling. Assuming that the temperature of the products in the full scale engine is the uniform temperature of the chamber volume is a good approximation. However, for the model chamber multiple problems are encountered. The operating conditions substantially differ with varying fuels and inert gas mass fractions. The flame temperature will vary under all of these conditions. The greater problem is that the chamber will experience a significant temperature gradient due to the placement of the single injector near the chamber wall. The temperature will be the dominating factor defining the speed of sound in this test facility. Experimentally determining the chamber temperature would be an improved technique for chamber sizing. In fact, the researchers at KARI experimentally determined the temperature in the model chamber. In this analytical approach, the results for the model chamber are near to the original size of the full scale engine. Therefore multiple chamber sizes have been designed, accounting for the cold gas region in the model chamber.

\section{Recommendations}

The secondary objective of this work is to improve the experimental technique. In order to improve the methodology, testing will be required. Through the design process, multiple improvements have been speculated. The suggested chamber sizing process seems insufficient for this single element test. A variable size chamber would allow a more simplistic approach to a test apparatus design. No assumptions would be required about the model chamber conditions. The variable width design also alleviates the need for fabricating multiple chambers when testing several injectors under various conditions. The variable width chamber would be rectangular in cross section with three fixed walls and a smoothly variable wall. This rectangular geometry would be compatible with the low pressure oscillation amplitude. The chamber could be tuned with the variable wall to match the full scale tangential frequency while testing. This chamber has an added advantage for simpler optical access through the flat walls. A high speed camera or chemiluminescence measurements would enhance the data collection to better understand the combustion phenomena.

The second improvement would be to instrument the apparatus with a multiplicity of high frequency pressure transducers. In accordance with NASA SP-194 ${ }^{9}$, multiple high pressure transducers will be placed in the chamber to distinguish spinning modes or combined modes of combustion instability. An added advantage beyond the designed apparatus would be gained from mounting transducers in the propellant manifolds.

The application of monopropellant injectors is an attractive idea. With the appropriate arrangement, a cluster of monopropellant injectors may be applicable. A portion of the fire face could be taken with monopropellant swirl injectors or impinging jets. Many configurations would be necessary to find a representative cluster that would demonstrate similarity. This scaling approach would be a subject of future research to determine accuracy. 


\section{Acknowledgments}

This work is sponsored by NASA under Contract NNM05AA22A. The authors would like to thank Tom Nesman and Jim Hulka of Marshall Space Flight Center and Jacobs Sverdrup, respectively for their technical advice and direction, also to Dr. Marlow Moser of the UAH Propulsion Research Center for input on the test facility design.

\section{References}

${ }^{1}$ Fisher, S. C., Dodd, F. E., and Jensen, R. J., "Scaling Techniques for Liquid Rocket Combustion Stability Testing," Progress in Astronautics and Aeronautics, Vol. 169, 1995. Liquid Rocket Engine Combustion Instability, ed. V. Yang, and W. E.

Anderson, AIAA.

${ }^{2}$ Penner, S. S., "On the Development of Rational Scaling Procedures for Liquid-Fuel Rocket Engines," Jet Propulsion, Feb. 1957, pp.156-68.

${ }^{3}$ Penner, S. S., and Fuhs, A. E., "On Generalized Scaling Procedures for Liquid-Fuel Rocket Engines," Combustion and Flame, Vol. 1, 1957, pp. 229-240.

${ }^{4}$ Dexter, C.E, Hulka, J. et al., "Scaling Techniques for Design, Development, and Test", Progress in Astronautics and Aeronautics Series, Vol. 200, 2004. Liquid Rocket Thrust Chamber: Aspects of Modeling, Analysis, and Design. ed.Yang, V., Habiballah, M. et al., AIAA.

${ }^{5}$ Lee, K., Seo, S., Song, J., Han, Y., Choi, H., Seol, W., "Combustion Stability Assessment of Double Swirl Coaxial Injectors Using Simulant Propellants," AIAA Paper 2005-4443, July 2005.

${ }^{6}$ Manski, D., et al., "Cycles for Earth-to-Orbit Propulsion," Journal of Propulsion and Power, Vol. 14, No. 5, 1998, pp. 588604.

${ }^{7}$ Vasin, A., et al., Otkrytoe Aktsioneroe Obschestvo "Nauchao-Proizvodatveabnoe Obiedianie", U.S. Patent Application for a "Liquid-propellant rocket engine chamber and its casing," 6,244,041, filed 10 Sept. 1999.

${ }^{8}$ Bazarov, V., Yang, V., Puri, P., "Chapter 2: Design and Dynamics of Jet and Swirl Injectors," in Progress in Astronautics and Aeronautics Series, Vol. 200, 2004. Liquid Rocket Thrust Chamber: Aspects of Modeling, Analysis, and Design. ed.Yang, V., Habiballah, M. et al., AIAA.

${ }^{9}$ Harrje, D. T., Reardon, F. H., "Liquid Propellant Rocket Combustion Instability", NASA SP-194, 1972.

${ }^{10}$ Bazarov, V. G., Liquid Injector Dynamics, Mashinostroenie, Moscow, 1979. 